\title{
HBG2 and HBG1 Nucleotide Substitutions and Hbf Production in Thalassemia Patients
}

\author{
Cristina Curcio $^{1 *}$, Benzoni $\mathrm{E}^{1}$, Seia $\mathbf{M}^{1}$ and Giannone $\mathrm{V}^{1}$ \\ ${ }^{1}$ Fondazione IRCCS Ca' Granda Ospedale Maggiore Policlinico, Italy
}

*Corresponding author: Cristina Curcio, Medical Genetics Laboratory, Fondazione IRCCS Ca' Granda Ospedale Maggiore Policlinico, Milan, Italy.

To Cite This Article: Cristina Curcio. HBG2 and HBG1 Nucleotide Substitutions and Hbf Production in Thalassemia Patients. Am J Biomed Sci \& Res. 2019 - 4(4). AJBSR.MS.ID.000802. DOI: 10.34297/AJBSR.2019.04.000802

Received: July 26, 2019 | Published: August 01, 2019

\begin{abstract}
$\mathrm{HbF}$ represents the main hemoglobin fraction during fetal life. After birth, the synthesis of $\mathrm{HbF}$ decreases gradually and is replaced by $\mathrm{HbA}$ so that $\mathrm{HbF}$ levels are less than $2 \%$ in adults. During adulthood, $\mathrm{HbF}$ may be slightly or significantly elevated because of pathological or nonpathological causes such as HPFH. This status is caused either by fetal globin gene promoter variants or large deletions affecting the human fetal globin genes. In thalassemia, an increase in $\mathrm{HbF}$ is linked to $\beta$-thalassemia major, $\delta \beta$-thalassemia or thalassemia intermedia and improves the clinical picture as $\gamma$ globin chains compensate for the lack of functional $\beta$ - globin chains. The degree of HbF persistence varies greatly among adults and is largely genetically controlled. Research of causes that induce the expression of $\gamma$ globin gene can impact the definition of clinical condition, as well as provide potential targets for treatment of hemoglobinopathy, as an increase in HbF production ameliorates $\beta$-thalassemia severity. In this report we present full DNA analysis of both HBG2 and HBG1 genes in a cohort of $96 \beta$ - and/or $\alpha$-thalassemia subjects with high HbF levels and 30 healthy individuals to update the list of HPFH polymorphisms.
\end{abstract}

Keywords: Fetal hemoglobin (HbF); Hereditary persistence of fetal hemoglobin (HPFH); $\gamma$ Globin genes; HPFH polymorphisms; Thalassemia; Hemoglobinopathy

Abbreviations: HbF: Fetal Hemoglobin; HbA: Adult Hemoglobin; HPFH: Hereditary Persistence of Fetal Hemoglobin; BCL11A: B cell CLL/lymphoma 11A; ZBTB7A: Zinc-Finger and BTB-domain-containing 7A; KLF1: Kruppel Like Factor 1; MLPA: Multiplex Ligation Probe Amplification; SNPs: Single Nucleotide Polymorphisms

\section{Introduction}

$\mathrm{HbF}$ is a high oxygen affinity tetramer consisting of two $\alpha$ and two $\gamma$ globin chains. Its role is to transfer oxygen from maternal to fetal circulation and represents the main hemoglobin fraction during fetal life. HbF production starts from the sixth week of gestation and replaces the embryonic hemoglobins Gower I, Gower II and Portland produced in the first weeks after conception and at birth it constitutes about $80 \%$ of total hemoglobin. After birth, the synthesis of $\mathrm{HbF}$ decreases gradually due to silencing of the expression of $\gamma$ globin genes and reciprocal increase in $\beta$-globin gene expression and is replaced by $\mathrm{HbA}$. In normal conditions only trace amounts of $\mathrm{HbF}$ (less than $2 \%$ ) are present in postnatal life after 1 year of age [1]. However, $\mathrm{HbF}$ may be slightly or significantly elevated during adulthood. A high level of $\mathrm{HbF}$ is mainly due to pathological conditions such as $\beta$-thalassemia major, $\delta \beta$-thalassemia, (as $\gamma$ globin chains compensate for the lack of functional $\beta$-globin chains) erythropoietic stress and bone marrow malignancies, or nonpathological conditions, known as HPFH, such as large deletions within the $\beta$-globin gene cluster (deletional HPFH), promoter variants of $\gamma$ globin genes (non deletional HPFH) or pregnancy [2-4]. While persistence of increased $\mathrm{HbF}$ production has no clinical consequences in healthy individuals, $\mathrm{HbF}$ is one of the most common and major modifiers of disease severity in individuals with $\beta$-thalassemia [58].

The degree of $\mathrm{HbF}$ persistence varies greatly among adults and is largely genetically controlled [9]. Gene expression is modulated by a large number of polymorphisms on cis-regulatory elements located between nucleotide -202 and -110 relative to the Cap site on promoter region of the HBG1 and HBG2 genes which disrupt transcription factor binding motif (BCL11A, ZBTB7A, MYB, KLF1) [10$12]$. The involvement of some variants has a clear association with increased $\mathrm{HbF}$ levels, although others are still ambiguous. Gene and genetic variant identification contributing to $\mathrm{HbF}$ variation and the molecular mechanisms through which they operate play an important role in diagnostic and prognostic medicine, as well as in research for novel therapeutic markers. In fact, the reactivation of $\mathrm{HbF}$ synthesis by several approaches, such as pharmacological 
and gene transfer, modulate the phenotype of patients with $\beta$-thalassemia [13]. The research of polymorphisms in $\gamma$ globin genes and direct or indirect transcriptional repressors of $\gamma$ globin gene expression has recently gained attention by aiding in the development of new therapeutic strategies and pharmacological agents that increase the levels of this hemoglobin [14]. In this report we present full DNA analysis of both HBG2 and HBG1 genes to update the list of HPFH polymorphisms.

\section{Materials and Methods}

We screened a cohort of $96 \beta$ - and/or $\alpha$-thalassemia subjects with high $\mathrm{HbF}$ levels who were referred to our diagnostic laboratory for ascertainment of possible hemoglobinopathy and 30 healthy individuals with $\mathrm{HbF}$ level $<2 \%$ recruited at Fondazione IRCCS $\mathrm{Ca}^{\prime}$ Granda, Ospedale Maggiore Policlinico in Milan. All subjects signed the informed consent form before blood sampling.

Patients were divided into 4 groups:

1. $8 \alpha$-thalassemia carriers (HbF level: $2.7 \%-32.6 \%$ )

2. $41 \beta$-thalassemia carriers (HbF level: $3 \%-50.3 \%$ )

3. $4 \beta$-thalassemia intermedia/major patients (HbF level: $67 \%-94.6 \%)$

4. $43 \gamma$-thalassemia patients (HbF level: $2.2 \%-16.5 \%)$

The hematological parameters were determined on Sysmex XN-9000, and hemoglobins were analyzed routinely by High Performance Liquid Chromatography- HPLC- Biorad D100, Biorad Laboratories, Hercules, CA, USA. Genomic DNA was extracted from peripheral blood samples. The research of polymorphisms in $\gamma$ globin genes was performed by Sanger sequencing (BigDye Terminator Cycle Sequencing Ready Reaction Kit v.1.1) on the ABI PRISM ${ }^{\circledR}$ 3130xl Genetic Analyzer (Applied Biosystems, Foster City, CA, USA). The research of deletional HPFH was performed by MLPA method according to the manufacturer's instructions (SALSA® MLPA ${ }^{\circledR}$ Probemix P102-D1 HBB; MRC-Holland, Amsterdam, The Netherlands).

\section{Results and Discussion}

Table $1 \& 2$ show the frequencies in patient and reference groups of HBG2 and HBG1 polymorphisms, respectively. HBB MLPA resulted negative for all analysed subjects (data not shown). In the $\beta$ - and $\alpha$-thalassemia carriers and $\beta$-thalassemia intermedia/major, an increased $\mathrm{HbF}$ level is due to their pathologic condition. In the $\beta$-thalassemia carrier group we found the c.227T $>C$ HbF-Sardinia $(\mathrm{A} \gamma \mathrm{T}$ ) variant together with $\beta 0$ c.118C $>\mathrm{T}$ (Codon 39 (C->T)) and an $\mathrm{HbF}$ increase of $10 \%$ of the total, as expected [15]. Two $\beta 0$ c. $92+1 \mathrm{G}>\mathrm{A}$ (IVS-I-1 (G->A)) patients present the c. $-170 \mathrm{G}>\mathrm{A}$ (rs35378915) mutation, called Greek HPFH, that affects the binding of three nuclear factors to the CCAAT region of the HBG1 gene (HbF: 10.3-50.3) [16]. In $\gamma$ - thalassemia patients, the high $\mathrm{HbF}$ level is not correlated to $\beta$ - and $\alpha$-thalassemia, even if we cannot exclude other malignancies. We identified the following SNPs exclusively in this group: rs73402643, rs531285196, rs904054277, rs757734616 and rs1231067410 in HBG2; rs35993903, rs34844625, rs3020750, rs2860456, rs1234411915, rs537552941, rs567305547, rs112286603 and c.*179G>A in HBG1. To note, c.*179G>A does not have an assigned reference SNP ID number.

\begin{tabular}{|c|c|c|c|c|c|}
\hline HBG2 POLYMORPHISMS (NM_000184.3) & $\begin{array}{l}\alpha \text {-thalassemia } \\
\text { carriers }\end{array}$ & $\begin{array}{l}\beta \text {-thalassemia } \\
\text { carriers }\end{array}$ & $\begin{array}{c}\beta \text {-thalassemia } \\
\text { intermedia/major } \\
\text { patients }\end{array}$ & $\begin{array}{c}\gamma \text { - thalassemia } \\
\text { patients }\end{array}$ & $\begin{array}{l}\text { Reference } \\
\text { group }\end{array}$ \\
\hline $\begin{array}{l}\text { c. }-558 \mathrm{C}>\mathrm{G} \text { (rs73402643) }(\mathrm{c} .-437-121 \mathrm{C}>\mathrm{G}) \\
475 \mathrm{bp} \text { before transcription start site }\end{array}$ & & & & $\begin{array}{c}1 / 86 \\
(\mathrm{f}=0.011)\end{array}$ & \\
\hline $\begin{array}{l}\text { c. }-450-445 \text { delCTTTAA (rs112075505) } \\
\text { 392-397 bp before transcription start site }\end{array}$ & $\begin{array}{c}1 / 16 \\
(\mathrm{f}=0.062)\end{array}$ & & & & \\
\hline $\begin{array}{c}\text { c. }-450 \mathrm{C}>\mathrm{T}(\mathrm{rs} 531285196) \\
397 \text { bp before transcription start site }\end{array}$ & & & & $\begin{array}{c}1 / 86 \\
(\mathrm{f}=0.011)\end{array}$ & \\
\hline $\begin{array}{c}\text { c. }-422 \mathrm{C}>\mathrm{G}(\mathrm{rs} 112215533) \\
369 \text { bp before transcription start site }\end{array}$ & & $\begin{array}{c}2 / 82 \\
(\mathrm{f}=0.024)\end{array}$ & & $\begin{array}{c}3 / 86 \\
(\mathrm{f}=0.034)\end{array}$ & \\
\hline $\begin{array}{c}\text { c. }-362 A>G(\text { rs } 112479156) \\
309 \text { bp before transcription start site }\end{array}$ & $\begin{array}{c}2 / 16 \\
(\mathrm{f}=0.125)\end{array}$ & $\begin{array}{c}2 / 82 \\
(\mathrm{f}=0.024)\end{array}$ & & $\begin{array}{c}5 / 86 \\
(\mathrm{f}=0.058)\end{array}$ & \\
\hline $\begin{array}{c}\text { c. }-324 \mathrm{~T}>\mathrm{C}(\mathrm{rs} 113622787) \\
271 \text { bp before transcription start site }\end{array}$ & $\begin{array}{c}1 / 16 \\
(f=0.062)\end{array}$ & & & & \\
\hline $\begin{array}{c}\text { c. }-321 \mathrm{G}>\mathrm{C}(\mathrm{rs} 904054277) \\
268 \mathrm{bp} \text { before transcription start site }\end{array}$ & & & & $\begin{array}{c}1 / 86 \\
(\mathrm{f}=0.011)\end{array}$ & \\
\hline $\begin{array}{c}\text { c. }-309 \mathrm{~A}>\mathrm{G} \text { (rs1045222350) } \\
256 \text { bp before transcription start site }\end{array}$ & $\begin{array}{c}1 / 16 \\
(\mathrm{f}=0.062)\end{array}$ & & & $\begin{array}{c}2 / 86 \\
(\mathrm{f}=0.023)\end{array}$ & \\
\hline $\begin{array}{c}\text { c. }-211 \mathrm{C}>\mathrm{T} \text { (rs7482144) } \\
158 \text { bp before transcription start site }\end{array}$ & $\begin{array}{c}6 / 16 \\
(f=0.375)\end{array}$ & $\begin{array}{c}19 / 82 \\
(\mathrm{f}=0.231)\end{array}$ & $\begin{array}{c}4 / 8 \\
(\mathrm{f}=0.500)\end{array}$ & $\begin{array}{c}29 / 86 \\
(\mathrm{f}=0.337)\end{array}$ & $\begin{array}{c}21 / 60 \\
(\mathrm{f}=0.350)\end{array}$ \\
\hline $\begin{array}{c}\text { c. }-69 \mathrm{C}>\mathrm{G} \text { (rs551623060) } \\
16 \text { bp before transcription start site }\end{array}$ & & $\begin{array}{c}1 / 82 \\
(\mathrm{f}=0.012)\end{array}$ & & & \\
\hline $\begin{array}{c}\text { c. } 93-58 \mathrm{C}>\mathrm{T}(\mathrm{rs} 1894398) \\
\text { intron } 1 \text { of } 2 \text { position } 65 \text { of } 122 \text { (intronic) }\end{array}$ & $\begin{array}{c}1 / 16 \\
(\mathrm{f}=0.062)\end{array}$ & $\begin{array}{c}22 / 82 \\
(\mathrm{f}=0.268)\end{array}$ & & $\begin{array}{c}9 / 86 \\
(\mathrm{f}=0.104)\end{array}$ & \\
\hline
\end{tabular}




\begin{tabular}{|c|c|c|c|c|c|}
\hline $\begin{array}{l}\text { c. } 315+24 \mathrm{~A}>\mathrm{C}(\mathrm{rs} 33993529) \\
\text { intron } 2 \text { of } 2 \text { position } 24 \text { of } 886 \text { (intronic) }\end{array}$ & $\begin{array}{c}1 / 16 \\
(\mathrm{f}=0.062)\end{array}$ & & & & \\
\hline $\begin{array}{c}\text { c.*18C>T (rs757734616) } \\
\text { exon } 3 \text { of } 3 \text { (3'UTR) position } 147 \text { of } 218\end{array}$ & & & & $\begin{array}{c}1 / 86 \\
(\mathrm{f}=0.011)\end{array}$ & \\
\hline $\begin{array}{l}\text { c.*54_*55ins A (rs34879481) } \\
\text { exon } 3 \text { of } 3 \text { (3'UTR) before position } 184 \text { of } 218\end{array}$ & $\begin{array}{c}6 / 16 \\
(f=0.375)\end{array}$ & $\begin{array}{c}18 / 82 \\
(f=0.219)\end{array}$ & $\begin{array}{c}1 / 8 \\
(\mathrm{f}=0.125)\end{array}$ & $\begin{array}{c}23 / 86 \\
(\mathrm{f}=0.267)\end{array}$ & $\begin{array}{c}21 / 60 \\
(\mathrm{f}=0.350)\end{array}$ \\
\hline $\begin{array}{c}\text { c. } * 160 \mathrm{G}>\mathrm{T}(\mathrm{rs} 1231067410) \\
71 \mathrm{bp} \text { after transcription end site }\end{array}$ & & & & $\begin{array}{c}1 / 86 \\
(\mathrm{f}=0.011)\end{array}$ & \\
\hline
\end{tabular}

\begin{tabular}{|c|c|c|c|c|c|}
\hline HBG1 POLYMORPHISMS (NM_000559.2) & $\begin{array}{l}\alpha \text {-thalassemia } \\
\text { carriers }\end{array}$ & $\begin{array}{l}\beta \text {-thalassemia } \\
\text { carriers }\end{array}$ & $\begin{array}{c}\beta \text {-thalassemia } \\
\text { intermedia/major } \\
\text { patients }\end{array}$ & $\begin{array}{c}\gamma \text { - thalassemia } \\
\text { patients }\end{array}$ & $\begin{array}{l}\text { Reference } \\
\text { group }\end{array}$ \\
\hline c. $-637 G>A(r s 2855039)$ & & $4 / 82(\mathrm{f}=0.048)$ & & $5 / 86(\mathrm{f}=0.058)$ & $\begin{array}{c}21 / 60 \\
(\mathrm{f}=0.350)\end{array}$ \\
\hline $\begin{array}{c}\text { c. }-548 \mathrm{~T}>\mathrm{A}(\mathrm{rs} 57966301) \\
495 \mathrm{bp} \text { before transcription start site }\end{array}$ & & $\begin{array}{c}1 / 82 \\
(\mathrm{f}=0.012)\end{array}$ & & $\begin{array}{c}2 / 86 \\
(\mathrm{f}=0.023)\end{array}$ & \\
\hline $\begin{array}{l}\text { c. }-447 \mathrm{G}>\mathrm{A}(\mathrm{rs} 35993903) \\
394 \mathrm{bp} \text { before transcription start site }\end{array}$ & & & & $\begin{array}{c}1 / 86 \\
(\mathrm{f}=0.011)\end{array}$ & \\
\hline $\begin{array}{l}\text { c. }-418 \mathrm{G}>\mathrm{C}(\mathrm{rs} 2855040) \\
365 \mathrm{bp} \text { before transcription start site }\end{array}$ & & $\begin{array}{c}1 / 82 \\
(\mathrm{f}=0.012)\end{array}$ & & $\begin{array}{c}2 / 86 \\
(\mathrm{f}=0.023)\end{array}$ & \\
\hline $\begin{array}{c}\mathrm{c}-405 \mathrm{~A}>\mathrm{G}(\mathrm{rs} 34844625) \\
352 \text { bp before transcription start site }\end{array}$ & & & & $\begin{array}{c}1 / 86 \\
(\mathrm{f}=0.011)\end{array}$ & \\
\hline $\begin{array}{c}\text { c. }-373 \mathrm{C}>\mathrm{T}(\mathrm{rs} 3020750) \\
320 \mathrm{bp} \text { before transcription start site }\end{array}$ & & & & $\begin{array}{c}1 / 86 \\
(\mathrm{f}=0.011)\end{array}$ & \\
\hline $\begin{array}{c}\text { c. }-366 \mathrm{~A}>\mathrm{G}(\mathrm{rs} 2860456) \\
313 \text { bp before transcription start site }\end{array}$ & & & & $\begin{array}{c}1 / 86 \\
(\mathrm{f}=0.011)\end{array}$ & \\
\hline $\begin{array}{c}\text { c. }-320 \mathrm{C}>\mathrm{T}(\mathrm{rs} 12290216) \\
267 \text { bp before transcription start site }\end{array}$ & $\begin{array}{c}1 / 16 \\
(\mathrm{f}=0.062)\end{array}$ & & & $\begin{array}{c}1 / 86 \\
(\mathrm{f}=0.011)\end{array}$ & \\
\hline $\begin{array}{l}\text { c.-275_-274 in sAGCA (rs561507744) } \\
222 \text { bp before transcription start site }\end{array}$ & $\begin{array}{c}10 / 16 \\
(\mathrm{f}=0.625)\end{array}$ & $\begin{array}{c}57 / 82 \\
(\mathrm{f}=0.695)\end{array}$ & $\begin{array}{c}4 / 8 \\
(\mathrm{f}=0.500)\end{array}$ & $\begin{array}{c}50 / 86 \\
(\mathrm{f}=0.581)\end{array}$ & $\begin{array}{c}51 / 60 \\
(\mathrm{f}=0.850)\end{array}$ \\
\hline $\begin{array}{l}\text { c. }-214 \mathrm{G}>\mathrm{A}(\mathrm{rs} 1234411915) \\
161 \text { bp before transcription start site }\end{array}$ & & & & $\begin{array}{c}1 / 86 \\
(\mathrm{f}=0.011)\end{array}$ & \\
\hline $\begin{array}{c}\text { c.-170G }>\mathrm{A}(\mathrm{rs} 35378915) \\
117 \text { bp before transcription start site }\end{array}$ & & $\begin{array}{c}2 / 82 \\
(\mathrm{f}=0.024)\end{array}$ & & & \\
\hline $\begin{array}{c}\text { c. }-69 \mathrm{C}>\mathrm{G} \text { (rs558015287) } \\
16 \text { bp before transcription start site }\end{array}$ & & $\begin{array}{c}1 / 82 \\
(\mathrm{f}=0.012)\end{array}$ & & & \\
\hline $\begin{array}{c}\text { c. }-29 \mathrm{G}>\mathrm{A}(\mathrm{rs} 368698783) \\
\text { exon } 1 \text { of } 3 \text { (5'UTR) position } 25 \text { of } 145\end{array}$ & $\begin{array}{c}3 / 16 \\
(\mathrm{f}=0.187)\end{array}$ & $\begin{array}{c}12 / 82 \\
(\mathrm{f}=0.146)\end{array}$ & $\begin{array}{c}2 / 8 \\
(\mathrm{f}=0.250)\end{array}$ & $\begin{array}{c}17 / 86 \\
(\mathrm{f}=0.197)\end{array}$ & $\begin{array}{c}21 / 60 \\
(\mathrm{f}=0.350)\end{array}$ \\
\hline $\begin{array}{l}\text { c. } 92+40 \mathrm{C}>\mathrm{A}(\mathrm{rs} 537552941) \\
\text { intron } 1 \text { of } 2 \text { position } 40 \text { of } 122 \text { (intronic) }\end{array}$ & & & & $\begin{array}{c}1 / 86 \\
(\mathrm{f}=0.011)\end{array}$ & \\
\hline $\begin{array}{l}\text { c. } 92+41 \mathrm{C}>\mathrm{A}(\mathrm{rs} 567305547) \\
\text { intron } 1 \text { of } 2 \text { position } 41 \text { of } 122 \text { (intronic) }\end{array}$ & & & & $\begin{array}{c}1 / 86 \\
(\mathrm{f}=0.011)\end{array}$ & \\
\hline $\begin{array}{l}\text { c. } 93-58 \mathrm{C}>\mathrm{T} \text { ( } \mathrm{rs} 202216517) \\
\text { intron } 1 \text { of } 2 \text { position } 65 \text { of } 122 \text { (intronic) }\end{array}$ & $\begin{array}{c}2 / 16 \\
(\mathrm{f}=0.125)\end{array}$ & $\begin{array}{c}26 / 82 \\
(\mathrm{f}=0.317)\end{array}$ & & $\begin{array}{c}12 / 86 \\
(\mathrm{f}=0.139)\end{array}$ & \\
\hline $\begin{array}{c}\text { c.227T >C p.Ile76Thr (rs1061234) } \\
\text { Hb F-Sardinia }(\mathrm{A} \gamma \mathrm{T})\end{array}$ & $\begin{array}{c}2 / 16 \\
(\mathrm{f}=0.125)\end{array}$ & $\begin{array}{c}8 / 82 \\
(f=0.097) \\
\end{array}$ & & $\begin{array}{c}4 / 86 \\
(f=0.046)\end{array}$ & \\
\hline $\begin{array}{l}\text { c.*3**6 delTCAC insCTCT (rs386750130) } \\
\text { exon } 3 \text { of } 3 \text { (3'UTR) position 132-135 of } 216\end{array}$ & $\begin{array}{c}11 / 16 \\
(\mathrm{f}=0.687)\end{array}$ & $\begin{array}{c}50 / 82 \\
(\mathrm{f}=0.609) \\
\end{array}$ & $\begin{array}{c}4 / 8 \\
(\mathrm{f}=0.500)\end{array}$ & $\begin{array}{c}49 / 86 \\
(\mathrm{f}=0.569)\end{array}$ & $\begin{array}{c}43 / 60 \\
(\mathrm{f}=0.716)\end{array}$ \\
\hline $\begin{array}{c}\text { c. } * 15 A>C \text { (rs112286603) } \\
\text { exon } 3 \text { of } 3 \text { (3'UTR) position } 144 \text { of } 216\end{array}$ & & & & $\begin{array}{c}1 / 86 \\
(\mathrm{f}=0.011)\end{array}$ & \\
\hline $\begin{array}{c}\text { c.*55delA (rs3841756) } \\
\text { exon } 3 \text { of } 3 \text { (3'UTR) position } 184 \text { of } 216\end{array}$ & $\begin{array}{c}1 / 16 \\
(f=0.062)\end{array}$ & $\begin{array}{c}15 / 82 \\
(\mathrm{f}=0.182)\end{array}$ & & $\begin{array}{c}10 / 86 \\
(\mathrm{f}=0.116)\end{array}$ & $\begin{array}{c}13 / 60 \\
(\mathrm{f}=0.216)\end{array}$ \\
\hline $\begin{array}{c}\text { c. } * 124 \mathrm{~T}>\mathrm{A}(\mathrm{rs} 2402330) \\
37 \mathrm{bp} \text { after transcription end site }\end{array}$ & & $\begin{array}{c}2 / 82 \\
(\mathrm{f}=0.024)\end{array}$ & & $\begin{array}{c}1 / 86 \\
(\mathrm{f}=0.011)\end{array}$ & \\
\hline c. ${ }^{*} 179 \mathrm{G}>\mathrm{A}$ & & & & $\begin{array}{c}1 / 86 \\
(\mathrm{f}=0.011)\end{array}$ & \\
\hline
\end{tabular}




\begin{tabular}{|c|c|c|c|c|}
\hline $\begin{array}{c}\text { c. } * 246 \mathrm{~A}>\mathrm{T}(\mathrm{rs} 916111) \\
159 \mathrm{bp} \text { after transcription end site }\end{array}$ & $\begin{array}{c}6 / 16 \\
(f=0.375)\end{array}$ & $\begin{array}{c}36 / 82 \\
(\mathrm{f}=0.439)\end{array}$ & $\begin{array}{c}22 / 86 \\
(f=0.255)\end{array}$ & $\begin{array}{c}30 / 60 \\
(\mathrm{f}=0.500)\end{array}$ \\
\hline $\begin{array}{c}\text { c. } * 259 \mathrm{~A}>\mathrm{T}(\mathrm{rs} 1143541) \\
172 \mathrm{bp} \text { after transcription end site }\end{array}$ & $\begin{array}{c}2 / 16 \\
(\mathrm{f}=0.125)\end{array}$ & $\begin{array}{c}2 / 82 \\
(\mathrm{f}=0.024)\end{array}$ & $\begin{array}{c}6 / 86 \\
(f=0.069)\end{array}$ & \\
\hline $\begin{array}{c}\text { c. } * 344 \mathrm{G}>\mathrm{A}(\mathrm{rs} 147256314) \\
257 \mathrm{bp} \text { after transcription end site }\end{array}$ & & & $\begin{array}{c}1 / 86 \\
(f=0.011)\end{array}$ & \\
\hline
\end{tabular}

In comparing patient and reference groups, nucleotide variations identified only in promoter regions of patient groups include rs73402643, rs112075505, rs531285196, rs112215533, rs112479156, rs113622787, rs904054277, rs1045222350 and rs551623060 in HBG2 and rs57966301, rs35993903, rs2855040, rs34844625, rs3020750, rs2860456, rs12290216, rs1234411915, rs35378915 and rs558015287 in HBG1. They are located between c. $-558 \mathrm{C}>\mathrm{G}$ (475bp before transcription start site) and c.-309A $>\mathrm{G}$ (256bp before transcription start site) in the HBG2 promoter region, and between c.-548T >A (495bp before transcription start site) and c.-366A $>\mathrm{G}$ (313bp before transcription start site) in the HBG1 promoter region.

Considering their position, frequency, absence in reference group and effect on $\mathrm{HbF}$ level (range from 2.5-5\%), these variations may modify transcription binding sites, resulting in up-regulation of $\gamma$ globin gene or influence the HBG2 and HBG1 ratio expression. Always considering patients versus reference group, we identified in the HBG2 intron: rs33993529 and the HBG1 intron: rs537552941, rs567305547 and rs1061234. The presence of c.9358C $>\mathrm{T}$ (rs1894398) in HBG2 and c.93-58C > T (rs202216517) in HBG1, are observed only inpatient groups, even if population frequencies, obtained from exomes and whole genomes studies, are $f=0.169(r s 202216517)$ and $f=0.425$ (rs1894398) [17]. Analysis of 3'UTR showed the presence of rs757734616 and rs1231067410 in HBG2 and rs112286603, rs2402330, c.*179G>A, rs1143541 and rs147256314 in HBG1. We identified the following SNPs in the promoter region in both patient and reference groups, with differing HbF levels (patient $>4 \%$ and reference $<2 \%$ ): HBG2: rs7482144 and rs34879481; HBG1: rs2855039, rs561507744, rs386750130, rs3841756 and rs916111.

Given their frequency in our cohort and the population frequency reported, we can assume they are polymorphisms. We cannot exclude that those SNPs induce HBG expression genes under erythropoietic stress conditions. Inducing erythropoietic stress in the reference group followed by $\mathrm{HbF}$ analysis could help define their role. We observed in all groups that a high frequency of HBG2 c.-211C>T (rs7482144) expressed concomitant with HBG1 c.-29G>A (rs368698783) is associated with a greater variability in $\mathrm{HbF}$ level (range from 0.4-15.1\%) [18]. Analysis of 3'UTR showed the presence of polymorphisms with a high frequency in both patient and reference groups, such as c.*54_*55insA (rs34879481) in HBG2 and c.*3_*6delTCACinsCTCT (rs386750130) in HBG1. Polymorphisms on 3'UTR may influence microRNAs binding, whose expression is enhanced/inhibited during erythroid differentiation and induction of $\mathrm{HbF}$ production [19]. Recent studies have revealed that some microRNAs influence the expression of the $\gamma$-globin gene, showing that high $\mathrm{HbF}$ levels in adulthood may result from a range of genetic factors which may explain the observed variations in $\mathrm{HbF}$ of healthy subjects and patients with hemoglobinopathies [20,21].

\section{Conclusion}

HPFH is a harmless condition characterized by a lack of changes in the synthesis of $\beta$-globin chains, resulting in an increase of $\gamma$ chains and consequently high $\mathrm{HbF}$ level [22]. However, slightly or severely increased $\mathrm{HbF}$ values in adults may hide a more complex clinical condition, such as $\beta$-thalassemia major, $\delta \beta$-thalassemia, or bone marrow malignancies. As known in literature, HBG2 and HBG1 promoter regions have a regulatory role in gene expression because they contain enhancer and silencer elements [23]. Our study proposes to list SNPs found in HBG2 and HBG1 promoter regions and in 3'UTR in a cohort of patients with hemoglobinopathies and subjects with slightly or severely increased $\mathrm{HbF}$ values and in a reference group, to underlie the importance of a correct ascertainment and interpretation of an elevated $\mathrm{HbF}$ level.

Our results show several SNPs found either in patient and reference groups, whose correlation to $\mathrm{HbF}$ expression isn't clear, although they might be involved in stimulation of HBG2 and HBG1 expression during stress erythropoiesis. Exclusively inpatient groups we have also identified nucleotide variations, which may play a role in transcription binding site modification and regulation of $\gamma$ globin genes expression. More data is necessary to define a genotype/phenotype correlation. Additional studies are necessary to clarify the possible role of identified SNPs in our cohort to better understand their impact on $\mathrm{HbF}$ production in adults and alleviation in thalassemia disease. The level of $\mathrm{HbF}$ is an important index in thalassemia because it can hide a complex genotype that could otherwise be misdiagnosed causing diagnostic errors and incorrect reproductive risk.

\section{Acknowledgements}

The authors would like to thank Martha Berliner (Harvey Medicine and Surgery Course - University of Pavia) for proofreading and editing the language of this minireview. The authors would like to acknowledge Fondazione G. e D. De Marchi Onlus - Milan - Italy for supporting this work.

\section{Conflict of Interest}

The authors declare no conflicts of interest. The necessary consent for publication was obtained from the patients' parents.

\section{References}

1. Amato A, Cappabianca P, Perri M, Zaghis I, Grisanti P, et al. (2014) Interpreting elevated fetal hemoglobin in pathology and health at the basic laboratory level: new and known $\gamma$-gene mutations associated with hereditary persistence of fetal hemoglobin. Int J Lab Hematol 36(1): 13-19. 
2. Hamid M, Mahjoubi F, Akbari MT, Arab A, Zeinali S, et al. (2009) Molecular analysis of $\gamma$-globin promoters, HS-111 and 3'HS1, in $\beta$-thalassemia intermedia patients associated with high levels of HbF. Hemoglobin 33(6): 428-438.

3. Craig JE, Barnetson RA, Prior J, Raven JL, Thein SL (1994) Rapid detection of deletions causing $\delta \beta$ thalassemia and hereditary persistence of fetal hemoglobin by enzymatic amplification. Blood 83(6):1673-1682.

4. Panyasai S, Fucharoen S, Surapot S, Fucharoen G, Sanchaisuriya K (2004) Molecular basis and hematologic characterization of $\delta \beta$-thalassemia and hereditary persistence of fetal hemoglobin in Thailand. Haematologica 89(7): 777-781.

5. Nagel RL, Platt OS (2001) General Pathophysiology of Sickle Cell Anemia In: Steinberg MH, Forget BG, Diggs DR and Nagel RL. (Eds) Disorders of Hemoglobin: Genetics, Pathophysiology and Clinical Management. Cambridge University Press, Cambridge.

6. Platt OS, Brambilla DJ, Rosse WF, Milner PF, Castro O, et al. (1994) Mortality in sickle cell disease. Life expectancy and risk factors for early death. N Engl J Med 330(23): 1639-1644.

7. Weatherall DJ, Clegg JB (2001) Inherited haemoglobin disorders: an increasing global health problem. Bull World Health Organ 79(8): 704712.

8. Mettananda S, Gibbons RJ, Higgs DR (2015) $\alpha$-Globin as a molecular target in the treatment of $\beta$-thalassemia. Blood 125(24): 3694-3701.

9. Garner C, Mitchell J, Hatzis T, Reittie J, Farrell M, et al. (1998) Haplotype mapping of a major QTL for foetal haemoglobin production on chromosome 6q23. Am J Hum Genet 62(6): 1468-1474.

10. Thein SL, Menzel S (2009) Discovering the genetics underlying foetal haemoglobin production in adults. Br J Haematol 145(4): 455-467.

11. Thein SL (2004) Genetic insights into the clinical diversity of $\beta$ - thalassemia. Br J Haematol 124(3): 264-274.

12. Chen Z, Luo HY, Basran RK, Hsu TH, Mang DWH, et al. (2008) A T-to-G transversion at nucleotide -567 upstream of HBG2 in a GATA-1 binding motif is associated with elevated Hemoglobin F. Mol Cell Biol 28: 43864393.
13. Garner CP, Tatu T, Best S, Creary L, Thein SL (2002) Evidence of genetic interaction between the $\beta$-blobin complex and chromosome $8 \mathrm{q}$ in the expression of fetal hemoglobin. Am J Hum Genet 70(3): 793-799.

14. Thein SL, Menzel S, Lathrop M, Garner C (2009) Control of fetal hemoglobin: new insights emerging from genomics and clinical implications. Hum Mol Genet 18(R2): R216-R223.

15. Masala B, Formato M, Manca L, Demuro P, Gallisai D, et al. (1986) Polymorphism of Foetal Haemoglobin in the Sardinian $\beta+-$ Thalassaemia. Acta Haematol 76(4): 208-211.

16. Superti-Furga G, Barberis A, Schaffner G, Busslinger M (1988) The -117 mutation in Greek HPFH affects the binding of three nuclear factors to the CCAAT region of the gamma-globin gene. EMBO J 7: 3099-3107.

17. Kopanos C, Tsiolkas V, Kouris A, Chapple CE, Albarca Aguilera M, et al. (2019) VarSome: The Human Genomic Variant Search Engine. Bioinformatics 35(11): 1978-1980.

18. Diyu C, Yangjin Z, Xinhua Z, Yuhua Y, Xiuqin B, et al. (2017) A Genetic Variant Ameliorates $\beta$-Thalassemia Severity by Epigenetic-Mediated Elevation of Human Fetal Hemoglobin Expression. Am J Hum Genet 101(1): 130-138.

19. Kosaka N, Sugiura K, Yamamoto Y, Yoshioka Y, Miyazaki H, et al. (2008) Identification of erythropoietin-induced microRNAs in haematopoietic cells during erythroid differentiation. Br J Haematol 142(2): 293-300.

20. Bianchi N, Zuccato C, Lampronti I, Borgatti M, Gambari R (2009) Expression of miR-210 during erythroid differentiation and induction of $\gamma$-globin gene expression. BMB Rep 42(8): 493-499.

21. Noh SJ, Miller SH, Lee T, Goh SH, Marincola FM, et al. (2009) Let-7 microRNA are developmentally regulated in circulating human erythroid cells. J Trans Med 7: 98.

22. Nussbaum RL, McInnes RR, Willard HF (2007) Genética médica. (7 $7^{\text {th }}$ edn) Rio de Janeiro: Guanabara koogan.

23. Elion J, Berg PE, Lapoumeroulie C, Trabuchet G, Mittelman M, et al. (1992) DNA sequence variation in a negative control region 5 ' to the beta-globin gene correlates with the phenotypic expression of the beta $\mathrm{s}$ mutation. Blood 79(3): 787-792. 